\title{
Evaluation of Cardiovascular Involvement and Cytokine Levels in Patients with Mucopolysaccharidosis
}

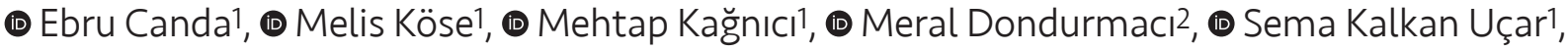 \\ (1) Eser Sözmen², (1) Reşit Ertürk Levent ${ }^{3}$, (1) Mahmut Çöker ${ }^{1}$ \\ ${ }^{1}$ Ege University Faculty of Medicine, Department of Pediatrics, Division of Metabolism and Nutrition, İzmir, Turkey \\ 2Ege University Faculty of Medicine, Department of Biochemistry, İzmir, Turkey \\ ${ }^{3}$ Ege University Faculty of Medicine, Department of Pediatrics, Division of Cardiology, İzmir, Turkey
}

\section{ABSTRACT}

Aim: Cardiovascular involvement is common in patients with mucopolysaccharidoses (MPS). In this study, we investigated the effects of the markers involved in vascular endothelial injury pathogenesis [transforming growth factor $\beta$ - (TCF- $\beta$ )], interleukin-6 (IL-6), IL-10, high sensitive-C reactive protein (hs-CRP), vascular endothelial growth factor (VEGF), N-terminal pro-Natriuretic peptide (NT-proBNP) and the clinical, laboratory and echocardiographic findings of the patients.

Materials and Methods: A total of 37 patients (5 MPS I, 4 MPS II, 2 MPS IIIa, 4 MPS IIIb, 14 MPS IVa, 8 MPS VI) and 32 controls with similar age and sex were included in the study.

Results: Corneal clouding was seen in 29 (78\%) patients. There were $23(62 \%)$ patients with organomegaly, and 28 (75\%) patients with hearing loss. When the groups were compared in terms of NT-proBNP, hs-CRP, TCF- $\beta$, IL- 6 , IL-10 and VEGF levels, there was a statistically significant increase in the patient group for NT-proBNP and VEGF ( $p=0.04, p=0.03$, respectively). The carotid intima media thickness was statistically significantly higher in the patient group $(p<0.001)$. The left ventricular diastolic diameter was significantly higher in the patient group ( $p=0.009)$, intraventricular septum thickness was significantly higher in the patient group $(p<0.001)$. The E/A ratio was significantly lower in the patient group $(p<0.001)$.

Conclusion: Cardiac involvement in MPS patients is a major cause of mortality and morbidity. It is thought that cytokines, proinflammatory markers are elevated in patients with vascular damage like other lysosomal diseases. There is a need for further studies to determine biomarkers for vascular involvement.

Keywords: Mucopolysaccharidoses, carotis intima media thickness, vascular involvement, cytokines

\section{Address for Correspondence}

Ebru Canda MD, Ege University Faculty of Medicine, Department of Pediatrics, Division of Metabolism and Nutrition, Izmir, Turkey Phone: +90 2322901245 E-mail: ebru.canda@gmail.com ORCID ID: orcid.org/0000-0002-9175-1998

Received: 07.10.2018 Accepted: 17.10.2018

${ }^{\oplus}$ Copyright 2019 by Ege University Faculty of Medicine, Department of Pediatrics and Ege Children's Foundation The Journal of Pediatric Research, published by Calenos Publishing House. 


\section{Introduction}

Mucopolysaccharidoses (MPS) are lysosomal storage disorders that occur when there is a deficiency of the enzymes responsible for the catabolism of glycosaminoglycans (GAG). Clinical symptoms develop as a result of the deposition of non-degraded GAGs in certain organs, as well as in all tissues. In total, seven types of MPS (I, II, III, IVA, VI, VII and IX) have been defined, relating to 11 different enzyme deficiencies. Its incidence is known to be 2-5 per 100.000 live births (1-3).

Cardiac pathologies may develop as a result of GAG deposition in the myocardium, cardiac valves and the myointima of the coronary arteries $(4,5)$. Severe valvular stenosis or failure may also occur due to the thickening and calcification of the mitral and aortic valves $(4,5)$. Cardiac involvement is more severe and occurs in the earlier stages of the disease in patients with Type I MPS and is a significant cause of mortality and morbidity in MPS patients. Histopathological investigations have identified GAG depositions in the cardiac valves, endocardium and myocardial coronary artery, and also in the aorta and conductive systems of MPS patients $(6,7)$. Heparan-, dermatan-, chondroitin- and keratan-sulfate based GAGs are found naturally in the structure of cardiac valves and in the giant vessels, although among these, dermatansulfate is the most common, meaning that cardiac valve involvement is common in patients with Type I, II and VI MPS (8). Cardiovascular involvement is common among MPS patients. While it may occur with all types of MPS, it more commonly accompanies and occurs in the earlier stages of the disease in MPS I, II and VI. That said, there is only limited data on the frequency of cardiac involvement in MPS VII, as MPS VII itself is a very rare condition $(9,10)$. Cardiac involvement may be seen in the form of cardiac valve thickening, left ventricular hypertrophy, coronary artery disease, rhythm disorders or other vascular involvements (11).

There is an increasing need to develop novel noninvasive means of predicting potential cardiovascular side effects. Several studies have identified a correlation between peripheral vascular endothelial dysfunction and coronary endothelial dysfunction, and the measurement of carotid artery thickness (C-IMT) is a useful method for the evaluation of peripheral vascular endothelium. In a study investigating the relationship between GAG metabolism disturbances and atherosclerosis, a complex process led by proteoglycans and involving a GAG metabolism was reported to have resulted in atherosclerosis and endothelial dysfunction (12). In the literature, histopathological similarities have been reported between the coronary artery lesions detected in an MPS I patient and the atherosclerotic changes observed in adults (13). An autopsy sampling of that patient showed that negative-loaded GAGs caused the activation of TGF- $\beta$ in the extracellular matrix of the myocardium, which may have led eventually to hypertrophic cardiomyopathy (14).

The present study makes a comprehensive evaluation of cardiac function and vascular endothelial involvement in patients followed-up with a diagnosis of MPS. To this end, we measured the levels of the markers; transforming growth factor $\beta$ (TGF $\beta$ ), interleukin-6 (IL-6), IL-10, high sensitive C-reactive protein (hs-CRP), vascular endothelial growth factor (VEGF), N-terminal-pro-Natriuretic peptide (NT-proBNP), involved in the pathogenesis of vascular endothelial damage, and investigated potential correlations between these levels and the clinical and laboratory findings of MPS patients, as well as obtaining information about peripheral vascular involvement based on C-IMT thickness and biochemical markers. The information collected in this study may provide clinicians with more treatment options for MPS patients, and the markers may also have potential for use during the follow-up of MPS patients.

\section{Materials and Method}

The study included MPS patients followed-up by the pediatric metabolism and nutrition outpatient clinics, and who also provided consent for participation in the study. The clinical and laboratory findings of the patients were retrieved from their medical records, while ECG and echocardiographs, as well as C-IMT measurements, were obtained as a routine part of patient follow-up in outpatient clinics. Data was collected retrospectively from medical records. Age- and gender-matched healthy individuals without any known chronic disorders were included in the study as a control group after providing informed consent for participation. C-IMT measurements of the controls were obtained. All blood samples were centrifuged and stored at $-20{ }^{\circ} \mathrm{C}$. TCF- $\beta$, IL-6, IL-10, hs-CRP, VEGF, and NT-proBNP levels were measured in these blood samples using ELISA kits.

A complete echocardiography evaluation by a padiatric cardiologist who was blinded to the diagnoses was performed in all patients with a two-dimensional M-Mode and Doppler Echocardiogram using a Vivid 9 system (GE Vinmed, Horten, Norway) M5Sc and 11L transducer. All patients were kept in a left decubitis position during examination and the measurements were made using techniques according to the recommendations of the American Society of Echocardiography (15). High-resolution B-mode ultrasonography was performed in all patients on the right carotid artery and C-IMT was calculated by taking the mean of three measurements. The details of the measurements were given in a previous study $(16,17)$.

The study was approved (approval number: 14-3.1/1 4.4.2014) by the Ethics Committee of Ege University Faculty of Medicine and was supported by Ege University Scientific Research Projects Coordination, İzmir, Turkey. 


\section{Statistical Analysis}

SPSS 22.0 for Windows was used for the statistical analysis. Qualitative data are presented as counts and percentages. Quantitative data were given as mean \pm standard deviation for normally distributed data or otherwise median and range. Student's t-test was used to compare the differences between the patient and control groups for normally distributed data. Mann-Whitney $U$ test was used for non-parametric parameters. For all comparisons, $p$ values lower than 0.05 were considered statistically significant.

\section{Results}

A total of 37 patients diagnosed with MPS were included in the study. The mean age of the patient group was $11.3 \pm 6.2$ years (2.5-35 years), while the mean age of the healthy control group was $12.3 \pm 6.0$ (3-36 years) years which consisted of 32 participants. The MPS patient group comprised 18 (48\%) females and 19 (52\%) males, while the healthy control group comprised 16 (50\%) female and $16(50 \%)$ male participants. The characteristics of the patients with MPS are detailed in Table I. The age and gender distribution within the two groups were not significantly different.

The study included a total of 37 patients diagnosed with MPS (5 MPS I, 4 MPS II, 2 MPS IIla, 4 MPS IIIb, 14 MPS IVa, 8 MPS VI) and 32 age- and gender-matched healthy controls. The majority of patients included in the study had been diagnosed with MPS IVa. Disease-related enzyme levels decreased, and urinary GAG excretion increased in all patients, and all patients had dysostosis multiplex findings. Corneal clouding was noted in 29 patients, and two patients received corneal transplants. In total, 23 (62\%) patients had organomegaly and 28 (75\%) patients had hearing loss. The systemic involvement of the patients with MPS is given in Figure 1. The most common location of cardiac involvement was the mitral valve in cases of single valve involvement. Simultaneous involvement of the mitral and aortic valves was present in 15 (47\%) patients and was most often seen as the co-involvement of the mitral and aortic valves (Figure 2). Echocardiographic investigations revealed normal findings in four patients, three of which had been diagnosed with MPS IA. Furthermore, four patients had increased left ventricular wall thickness and left ventricular dysfunction; and one patient experienced a marked pulmonary gradient increase and an enlargement of the right cardiac structure.

In the patient group, the median NT-proBNP level was 44.4 (range: $17.6-495$ ) $\mathrm{pg} / \mathrm{mL}$, the median VEGF level was 518.5 (range: 95-6359) ng/L. In the healthy control group, the median NT-proBNP level was 29.2 (range: 19.6-81.53) $\mathrm{pg} / \mathrm{mL}$, the median VEGF level was 235.0 (range: 90.01796) ng/L. When the NT-proBNP, hs-CRP, TCF- $\beta$, IL-6, IL-10 and VEGF levels were compared between the two groups, significant elevations were noted for NT-proBNP and VEGF in the patient group when compared to the healthy controls ( $p=0.04, p=0.03$, respectively) (Table II). Cytokine levels of the patients and control group are given in Table I.

The patients were further classified into two groups, group 2 [(MPS I, II, IV and VI $(n=29)$ ], which underwent enzyme therapy and group 1, which underwent no enzyme therapy [(MPS IVA-2 patient-pre-treatment and diagnosed with MPS III $(n=6)]$. NT-proBNP, hs-CRP, TCF- $\beta, I L-6$, and IL-10 levels were not found to be significantly different between the patients who were receiving enzyme therapy and those who were not (Table III).

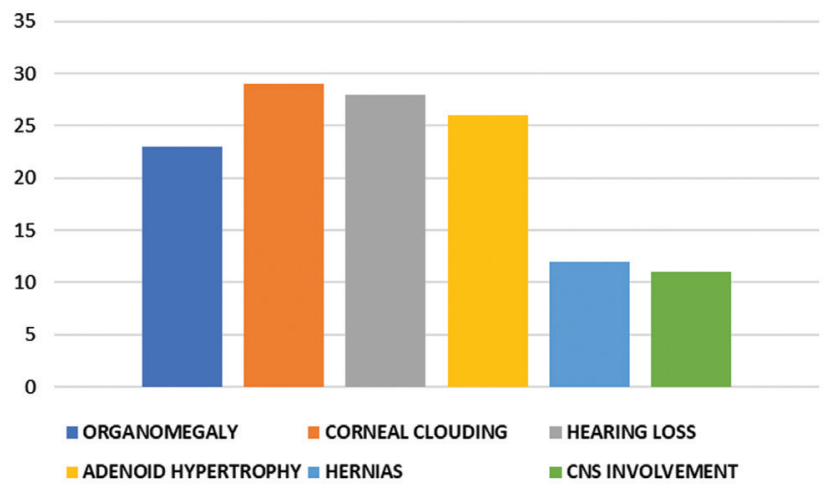

Figure 1. Clinical findings of the patients

\begin{tabular}{|l|l|l|l|l|l|}
\hline \multicolumn{6}{|c|}{ Table I. Characteristics of the patients with mucopolysaccharidosis } \\
\hline MPS type & Number & Gender, (M/F) & $\begin{array}{l}\text { Age (years), (min- } \\
\text { max) }\end{array}$ & $\begin{array}{l}\text { Age at diagnosis (years), (min- } \\
\text { max) }\end{array}$ & Alive / treatment with ERT \\
\hline I & 5 & $2 / 3$ & $8.7 \pm 5.8(2.5-16.5)$ & $4.2 \pm 6.0(0.9-15)$ & $4 / 4$ \\
\hline II & 4 & $4 /-$ & $8.6 \pm 4.2(4-13.5)$ & $3.1 \pm 1.7(1.0-5.0)$ & $3 / 3$ \\
\hline IIIa & 2 & $2 /-$ & 9 and 12.5 & 2 and 4 & - \\
\hline IIIb & 4 & $2 / 2$ & $13.7 \pm 1.8(12-16)$ & $6.0 \pm 2.0(3-7)$ & $1 /-$ \\
\hline IV & 14 & $6 / 8$ & $12.1 \pm 8.4(4.3-35)$ & $7.6 \pm 8.5(0.5-32)$ & $12 / 11$ \\
\hline VI & 8 & $4 / 4$ & $11.8 \pm 4.4(6.5-17)$ & $5.1(2.2) 2.5-9.0$ & $7 / 7$ \\
\hline
\end{tabular}

M: Male, F: Female, min: Minimum, max: Maximum, ERT: Enzyme replacement therapy, MPS: Mucopolysaccharidosis 
The carotid intima media thickness was significantly higher in the patient group when compared to the controls $(p<0.001)$ (Figure 3), and the left ventricular diastolic

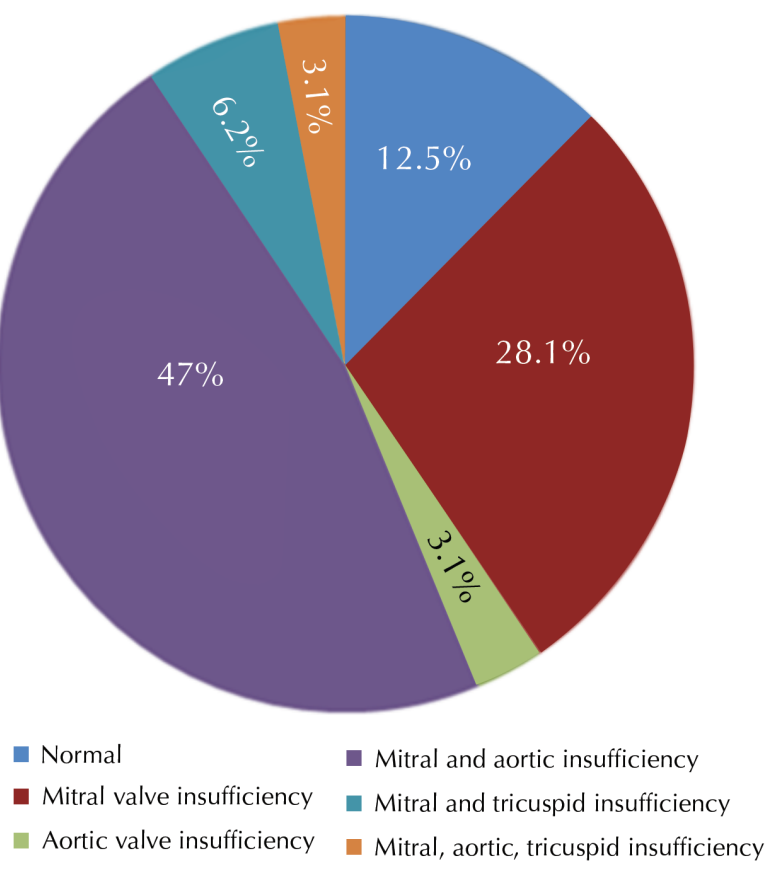

Figure 2. Valvular involvement of the patients diameter was also significantly higher in the patient group $(p=0.09)$. Intraventricular septum thickness was significantly higher in the patient group $(p<0.001)$. The $E / A$ ratio was significantly lower in the patient group $(p<0.001)$. The right ventricular end-diastolic diameter (RVd) was also higher in the patient group than in the controls, although not to a statistically significant degree $(p=0.05)$. The right ventricular end-systolic diameter was significantly higher in the patient group $(p<0.001)$. The echocardiographic evaluations results of the patients are given in Table IV.

\section{Discussion}

In our study, we evaluated proinflammatory cytokine levels in different types of MPS and compared with healthy controls. Progressive valvular involvement is the most common cardiac pathology among MPS patients (60-90\%) (14), and dysfunction due to the thickening of the cardiac valves has been reported previously in $80 \%$ of MPS I patients by Pastores et al. (18), and in 57\% of MPS II patients (19). Several studies have shown that the mitral valve was the most commonly involved valve, and valve deficiency was more common than obstruction (4). In their study of MPS Type VI patients, Azevedo et al. (20) reported that the mitral valve was the most common location of cardiac involvement, with mitral failure noted in more than $95 \%$ of their patients. The mitral valve was also the most commonly

Table II. Cytokine levels of the patient and control group

\begin{tabular}{|l|l|l|l|}
\hline & Patients median (range) & Control median (range) & p value \\
\hline NT-proBNP $(\mathrm{pg} / \mathrm{mL})$ & $44.4(17.6-495.0)$ & $29.2(19.6-81.53)$ & 0.04 \\
\hline Hs-CRP $(\mathrm{mg} / \mathrm{L})$ & $7.0(2.6-75)$ & $4.8(2.5-26.6)$ & 0.37 \\
\hline TGF- $\beta$ (ng/mL) & $160.6(76-2079.0)$ & $109.0(78.6-480.3)$ & 0.65 \\
\hline IL-6 $(\mathrm{ng} / \mathrm{L})$ & $85.8(41.0-995.0)$ & $72.5(45.7-237)$ & 0.66 \\
\hline VEGF $(\mathrm{ng} / \mathrm{L})$ & $518.5(95-6359)$ & $235.0(90.0-1796.0)$ & 0.03 \\
\hline IL-10 $(\mathrm{pg} / \mathrm{mL})$ & $340.5(91-3435)$ & $203.5(111.0-881.0)$ & 0.45 \\
\hline
\end{tabular}

NT-proBNP: N-terminal pro-natriuretic peptide, hs-CRP: High sensitive C-reactive protein, TGF- $\beta$ : Transforming growth factor- $\beta$, IL: Interleukin, VECF: Vascular endothelial growth factor

Table III. Cytokine levels of the treated and untreated patients

\begin{tabular}{|l|l|l|l|}
\hline & Untreated patients median (range) & Treated patients median (range) & p value \\
\hline Number of Patients & 8 & 29 & - \\
\hline NT-proBNP $(\mathrm{pg} / \mathrm{mL})$ & $42.8(91-3435)$ & $44.4(17-495)$ & 0.8 \\
\hline Hs-CRP $(\mathrm{mg} / \mathrm{L})$ & $5.3(2.9-53)$ & $7.3(2.6-75)$ & 1 \\
\hline TGF- $\beta$ (ng/mL) & $155(86.8-866.0)$ & $162.1(76.8-2079)$ & 0.8 \\
\hline IL-6 $(\mathrm{ng} / \mathrm{L})$ & $77.6(54.2-494.7)$ & $89.1(41.0-995.0)$ & 1 \\
\hline VEGF $(\mathrm{ng} / \mathrm{L})$ & $633.5(116-4699)$ & $467.0(95-6359)$ & 0.7 \\
\hline IL-10 $(\mathrm{pg} / \mathrm{mL})$ & $253.5(151-1461)$ & $354.5(91-3435)$ & 0.9 \\
\hline
\end{tabular}

NT-proBNP: N-terminal pro-natriuretic peptide, hs-CRP: High sensitive C reactive protein, TGF- $\beta$ : Transforming growth factor- $\beta$, IL: Interleukin, VEGF: Vascular endothelial growth factor 


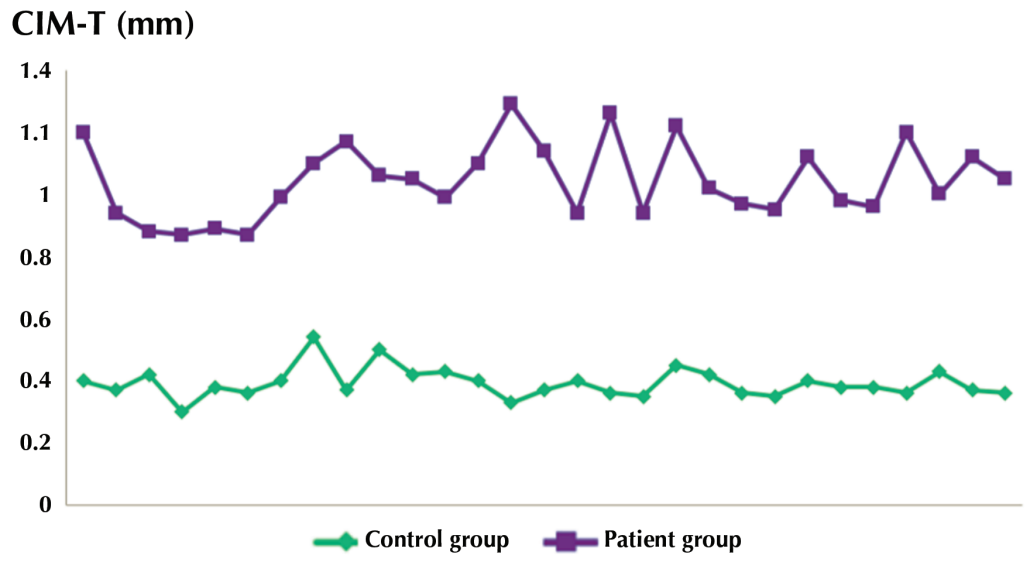

Figure 3. C-IMT levels of the patients and control groups C-IMT: Carotid artery thickness

\begin{tabular}{|l|l|l|l|}
\hline \multicolumn{1}{|l|}{ Table IV. Cardiac evaluation of the patients with echocardiography } \\
\hline & Patients mean (min-max) & Control mean (min-max) & p value \\
\hline Heart rate (minimum) & $109.6 \pm 19.4(75-163)$ & $81 \pm 9.7(65-105)$ & $<0.001$ \\
\hline LVDd & $32.7 \pm 5.58(23.1-44.0)$ & $29.6 \pm 2.91(23.7-37.8)$ & 0.009 \\
\hline LVDs & $19.1 \pm 5.4(12.1-31.8)$ & $18.8 \pm 2.09(14.5-22.6)$ & 0.7 \\
\hline RVDd & $18.5 \pm 4.1(6.5-24.5)$ & $16.9 \pm 1.3(15-20)$ & 0.05 \\
\hline RVDs & $13.6 \pm 2.6(9.5-19.6)$ & $11.1 \pm 1.8(7.8-15.6)$ & $<0.001$ \\
\hline IVSDd & $6.7 \pm 2.3(4.2-16.5)$ & $3.7 \pm 0.5(2.9-5)$ & $<0.001$ \\
\hline E/A ratio & $1.1 \pm 0.30 .6-2.1)$ & $1.5 \pm 0.1(1.2-2)$ & $<0.001$ \\
\hline
\end{tabular}

Min: Minimum, Max: Maximum, LVDd: Left ventricular diameter in diastole, LVDs: Left ventricular diameter in systole, RVDd: Right ventricular diameter in diastole, RVDs: Right ventricular diameter in systole, IVSDd: Intraventricular diameter in diastole

involved valve in the present study. While 15 patients had mitral and aortic valve involvement, four had increased left ventricular size and dysfunction, and one patient had a marked pulmonary gradient increase and enlarged right cardiac structures.

C-IMT is a useful method for the evaluation of peripheral vascular endothelium. In another study, Wang et al. (21) compared the C-IMT of MPS patients and healthy controls, evaluating 16 MPS patients based on neck C-IMT measurements and echocardiographic evaluations. The widths of the left main coronary left anterior descending and right coronary artery were not significantly different when compared to the control group, although mitral valve and aortic failure, as well as left ventricular dilatation, were found to be significantly more common in the MPS patients than in the control group. C-IMT levels were also elevated in MPS patients compared to the controls. The authors underlined the need for future studies investigating the correlation between C-IMT and arterial elasticity, and potential biomarkers for vascular dysfunction (12). In the present study, the C-IMT levels in the patient group were significantly higher than in the healthy controls.
In the literature, histopathological similarities have been reported between the coronary artery lesions detected in a MPS I patient and the atherosclerotic changes observed in adults. An autopsy sampling of that patient showed that negative-loaded GAGs caused the activation of TGF- $\beta$ in the extracellular matrix of the myocardium, which may have led eventually to hypertrophic cardiomyopathy (13). In a study carried out by Yano et al. (22) on an autopsy specimen of a deceased MPS I patient, "phosphorylated Smad 2" immunofluorescent staining revealed a significant signal increase in the vascular wall and the myocardium when compared to the control samples. This supports the increased TGF- $\beta$ signal (22). In another study investigating the relationship between TGF- $\beta$ and arterial pathology in MPS I animal models, immunohistochemically investigations of the sclerotic vessels showed TGF- $\beta$ positivity and an increase in fibronectin (23). According the literature findings, we expected to find a difference between the groups for TGF- $\beta$ levels, but we could not find any significant difference for TGF- $\beta$ levels between the groups. However, the levels were higher in the patient group. With the investigation 
of a larger number of patients, we think that a significant difference may be detected.

VEGF is a family of multifunctional growth factors that may have specific effects particularly on the endothelial cells. The family has six sub-members that play roles in the proliferation, migration and differentiation of endothelial cells. The expression of VEGF increases following hypoxia associated with an obstruction of the cardiac vessels (24). The relationship between VEGF and obstructions of the coronary or peripheral arteries has to date not been investigated specifically in MPS patients. An earlier study investigated the role of VEGF in fabry disease, which is another lysosomal storage disorder, and VECF levels were found to be elevated in patients with fabry disease. Based on their results, the authors of the study underlined that VEGF may be developed as a response to vascular damage in lysosomal diseases (25). There have been no studies to date investigating VECF levels in MPS patients. In the present study, we found that VEGF levels were significantly elevated in patients diagnosed with MPS.

Proinflammatory cytokines IL-6 and hs-CRP play a role in atherosclerotic plaque destabilization (26). A study involving 279 patients with atherosclerosis showed that hs-CRP, IL-6 and IL-10 were associated with C-IMT levels in atherosclerosis, and C-IMT and NT-proBNP were also associated with sudden cardiac events (27). In a study performed by Krecki et al. (28), hs-CRP and NT-proBNP levels were found to rise in proportion to the severity of the disease in patients with coronary artery atherosclerosis. Donida et al. (29) investigated oxidative stress and inflammation markers in 17 patients with MPS Type IVA who were undergoing enzyme replacement therapy and found that IL-6 levels were higher in the MPS IVA patients than in the healthy controls. In our study, we detected a statistical difference between groups for NT-proBNP levels. The levels of hs-CRP, IL- 6 and IL-10 were all higher in MPS patients than in the control group but there was no difference between groups.

The levels of the biomarkers investigated in this study were found to be elevated in MPS patients. Only NT-proBNP and VEGF levels were statistically different. C-IMT was also significantly elevated. These findings could be due to the inflammatory processes atherosclerosis developed in MPS patients. To the best of our knowledge, cytokines and proinflammatory markers have not been evaluated together in different types of MPS patients.

\section{Conclusion}

Cardiovascular involvement is a significant cause of mortality and morbidity in MPS patients. As is the case with other lysosomal diseases, MPS patients can be expected to have increased levels of cytokines and proinflammatory markers associated with vascular damage. The levels of biomarkers investigated in the present study were found to be elevated in MPS patients. Additional studies are required to investigate the potential use of markers of vascular involvement during the follow-up of MPS patients. In line with the earlier studies with similar findings, new treatment options need to be considered that suppress the inflammatory process induced by deposition.

\section{Ethics}

Ethics Committee Approval: The study was approved by the ethics comity of Ege University Faculty of Medicine (approval number:14-3.1/1 4.4.2014).

Informed Consent: Consent form was filled out by all participants.

Peer-review: External and internal peer-reviewed.

\section{Authorship Contributions}

Surgical and Medical Practices: E.C., R.E.L., E.S., M.D., Concept: E.C., M.Ç., S.K.U., Design: E.C., M.Ç., Data Collection or Processing: E.C., M.K., M.D., Analysis or Interpretation: E.C., M.Ç., S.K.U., E.S., Literature Search: E.C., M.K., M.KA., Writing: E.C.

Conflict of Interest: No conflict of interest was declared by the authors.

Financial Disclosure: This study supported by Ege University Scientific Research Projects Coordination, İzmir, Turkey.

\section{References}

1. Wraith JE. The mucopolysaccharidoses: a clinical review and guide to management. Arch Dis Child 1995;72:263-7.

2. Ferrari S, Ponzin D, Ashworth $\mathrm{L}$, et al. Diagnosis and management of ophthalmological features in patients with mucopolysaccharidosis. Br J Ophthalmol 2011;95:613-9.

3. Lachman R, Martin KW, Castro S, Basto MA, Adams $A$, Teles EL. Radiologic and neuroradiologic findings in the mucopolysaccharidoses. J Pediatr Rehabil Med 2010;3:109-18.

4. Braunlin EA, Harmatz PR, Scarpa M, et al. Cardiac disease in patients with mucopolysaccharidosis: presentation, diagnosis and management / Inherit Metab Dis 2011;34:1183-97.

5. Braunlin EA, Berry JM, Whitley CB. Cardiac findings after enzyme replacement therapy for mucopolysaccharidosis Type I. Am I Cardiol 2006;98:416-8.

6. Rentería VG, Ferrans VJ, Roberts WC. The heart in the Hurler syndrome: gross, histologic and ultrastructural observations in five necropsy cases. Am J Cardiol 1976;38:487-501.

7. Hishitani T, Wakita S, Isoda T, Katori T, Ishizawa A, Okada R. Sudden death in Hunter syndrome caused by complete atrioventricular block. J Pediatr 2000;136:268-9.

8. Latif N, Sarathchandra P, Taylor PM, Antoniw I, Yacoub MH. Localization and pattern of expression of extracellular matrix components in human heart valves. I Heart Valve Dis 2005;14:218-27.

9. Chen $\mathrm{YL}, \mathrm{Wu} \mathrm{KH}$. Airway management of patients with craniofacial abnormalities: 10-year experience at a teaching hospital in Taiwan. J Chin Med Assoc. 2009;72:468-70. 
10. Dangel $\mathrm{H}$. Cardiovascular changes in children with mucopolysaccharide storage diseases and related disordersclinical and echocardiographic findings in 64 patients. Eur J Pediatr 1998;157:534-8.

11. Brands $M$ M, Frohn-Mulder IM, Hagemans $M L$, et al. Mucopolysaccharidosis: cardiologic features and effects of enzyme-replacement therapy in 24 children with MPS I, II and VI. J Inherit Metab Dis 2013;36:227-34.

12. Wang RY, Covault KK, Halcrow EM, et al. Carotid intima-media thickness is increased in patients with mucopolysaccharidosis. Mol Genet Metab 2011;104:592-6.

13. Yano S, Moseley K, Wong L, Castelnovi C, Azen C, Pavlova Z. Glycosaminoglycan metabolism defects and atherosclerosis: frequent association of endothelial dysfunction in patients with Mucopolysaccharidosis. J Inherit Metab Dis 2014;37:255-61.

14. Wippermann CF, Beck M, Schranz D, Huth R, Michel-Behnke I, Jüngst BK. Mitral and aortic regurgitation in 84 patients with mucopolysaccharidoses. Eur / Pediatr 1995;154:98-101.

15. Sahn DJ, DeMaria A, Kisslo J, Weyman A. Recommendations regarding quantitation in $M$-mode echocardiography: results of a survey of echocardiographic measurements. Circulation 1978; 58:1072-83.

16. Levent E, Gökşen D, Ozyürek AR, Darcan S, Coker M. Usefulness of the myocardial performance index (MPI) for assessing ventricular function in obese pediatric patients. Turk J Pediatr 2005;47:34-8.

17. Touboul PJ, Hennerici MG, Meairs S, et al. Mannheim carotid intima-media thickness consensus (2004-2006). An update on behalf of the Advisory Board of the $3^{\text {rd }}$ and $4^{\text {th }}$ Watching the Risk Symposium, 13th and 15th European Stroke Conferences, Mannheim, Germany, 2004, and Brussels, Belgium, 2006. Cerebrovasc Dis 2007;23:75-80.

18. Pastores GM, Arn P, Beck M, et al. The MPS I registry: design, methodology, and early findings of a global disease registry for monitoring patients with Mucopolysaccharidosis Type I. Mol Genet Metab 2007;91:37-47.

19. Wraith JE, Beck M, Giugliani R, et al. Initial report from the Hunter Outcome Survey. Genet Med 2008;10:508-16.
20. Azevedo AC, Schwartz IV, Kalakun L, et al. Clinical and biochemical study of 28 patients with mucopolysaccharidosis type VI. Clin Genet. 2004;66:208-13.

21. Wang RY, Covault KK, Halcrow EM, et al. Carotid intima-media thickness is increased in patients with mucopolysaccharidosis. Mol Genet Metab 2011;104:592-6.

22. Yano S, Li C, Pavlova Z. The transforming growth factor-beta signaling pathway involvement in cardiovascular lesions in Mucopolysaccharidosis-I. JIMD Rep 2013;7:55-8.

23. Lyons JA, Dickson PI, Wall JS, et al. Arterial pathology in c anine mucopolysaccharidosis-I and response to therapy. Lab Invest 2011;91:665-74.

24. Yazir $Y$, Gonca S, Filiz S, Dalçık H. An important protein family for endothelial cells; vascular endothelial growth factor (Vegf) members of the family, structure and synthesis. C.Ü.Tıp Fakültesi Dergisi 2004;26:181-4.

25. Zampetti A, Gnarra M, Borsini W, et al. Vascular endothelial growth factor (VEGF-a) in fabry disease: Association with cutaneous and systemic manifestations with vascular involvement. Cytokine 2013;61:933-9.

26. Naghavi M, Falk E, Hecht HS, et al. From vulnerable plaque to vulnerable patient. Part III. Executive summary of the screening for heart attack prevention and education (SHAPE) task force report. Am J Cardiol 2006;98:2-15.

27. Kabłak-Ziembicka A, Przewłocki T, Stępień E, et al. Relationship between carotid intima-media thickness, cytokines, atherosclerosis extent and a two-year cardiovascular risk in patients with arteriosclerosis. Kardiol Pol 2011;69:1024-31.

28. Krecki $R$, Drozdz I, Szcześniak P, Orszulak-Michalak $D$, Krzemińska-Pakuta M. Novel atherogenesis markers for identification of patients with a multivessel coronary artery disease. Kardiol Pol 2008;66:1173-80.

29. Donida B, Marchetti DP, Biancini GB, et al. Oxidative stress and inflammation in mucopolysaccharidosis Type IVA patients treated with enzyme replacement therapy. Biochim Biophys Acta 2015;1852:1012-9. 